\title{
Compost Amended Media and Irrigation System Influence Containerized Perennial Salvia
}

\author{
S.B. Wilson and P.J. Stoffella \\ Indian River Research and Education Center, Institute of Food and Agricultural Sciences, University of \\ Florida, 2199 South Rock Road, Fort Pierce, FL 34945 \\ D.A. Graetz \\ Soil and Water Science Department, Institute of Food and Agricultural Sciences, 106 Newell Hall, \\ University of Florida, Gainesville, FL 32611-0510
}

AdDitional InDEX words. Salvia, biosolids, subtropical plant production, ornamentals, ebb and flow

\begin{abstract}
Three perennial species, wine sage (Salvia spp. Sell x roenen Schultes 'Van Houttei'), blue anise sage (Salvia gauranitica St.-Hil. Ex Benth. 'Black and Blue'), and indigo spires salvia (S. longispicata Martius Galeotti x S. farinacea Benth. 'Indigo Spires') were transplanted in containers filled with a biosolid-yard waste compost, a commercial peatbased mix, or a mixture of 1 compost : 1 peat-based mix by volume) and grown under ebb-and-flow, drip, or manual irrigation systems. Initial physical, chemical, and elemental analyses of the media indicated that compost alone had higher pH, electrical conductivity (EC), total porosity (TP), bulk density (BD), particle density (PD), N, C, P, Ca, Zn, Cu, Fe, and $\mathrm{B}$; lower initial moisture, $\mathrm{Mg}$ and $\mathrm{Al}$; and similar Mn contents than did the $100 \%$ peat-based medium. Heavy metal (Cd and $\mathrm{Pb}$ ) contents of compost did not exceed EPA 503 Rule limits for biosolid usage. After 6 weeks, plants were measured for leaf nutrient content, growth (leaf and stem dry weights, stem lengths), and quality (number of flowers, leaf greenness, and subjective quality ratings). At 6 weeks, plants grown in $50 \%$ or $100 \%$ compost generally had higher leaf $\mathrm{K}$, $\mathrm{P}$, and $\mathrm{Mn}$; similar $\mathrm{N}$ and $\mathrm{Ca}$; and lower $\mathrm{Mg}$, Fe, and Al content than plants grown in the $100 \%$ peat-based medium. Plants grown in media amended with compost generally produced similar or slightly smaller plants (stem weight, leaf weight, and stem length) than when grown in peat-based media. Plants irrigated by ebb and flow resulted in higher ('Van Houttei') or similar ('Indigo Spires') dry stem weights than plants irrigated manually or with drip irrigation. Plants grown in compost had leaf SPAD readings (leaf greenness), number of flowers, and visual quality ratings that were generally similar ('Indigo Spires') or slightly reduced ('Van Houttei') than plants grown in peat-based media. However, for each species (except for 'Van Houttei' grown in $50 \%$ compost using drip irrigation), plants were of marketable quality, regardless of irrigation system or medium. This study suggests that compost may serve as a viable alternative substrate for peat in the production of containerized perennials using ebb-and-flow, manual, or drip irrigation systems.
\end{abstract}

The nursery industry uses peat extensively as a primary component in commercial soilless potting media due to its organic composition and superior water holding capacity. Both environmental and economical implications of peat usage have resulted in the development of new substrate substitutes worldwide, most of which use waste byproducts. Mandated processing of waste by-products in the United States has inspired national interest in addressing the effectiveness of using composted biosolids and yard trimmings to grow containerized plants. Fitzpatrick (2001) has reviewed and cited numerous investigations illustrating the beneficial effect of compost utilization in greenhouse and nursery crop production systems. Composts can provide a valuable source of nutrients within a nursery substrate (Hue and Sobieszcyk, 1999). In addition, composts have soilborne disease suppression properties (Hoitink et al., 1991) and generally improve the physical, chemical, and biological properties of substrates (Inbar et al., 1993). In our previous studies, the effects of compost-amended media on the growth of perennials were investigated (Wilson et al., 2001, 2002). Several herbaceous perennial plant species, such as mexican heather (Cuphea hyssopifolia H.B.K.), Bolivian sunset [Gloxinia sylvatica (HBK) Wiehler], Brazilian plume (Justicia carnea Lindl.), and golden globe (Lysimachia congestiflora), were grown in commercial

Received for publication 21 May 2002. Accepted for publication 12 Nov. 2002. Florida Agricultural Experiment Station journal series R-08582. Authors gratefully acknowledge Laurie Krumfolz and Dawn Lucas for their technical assistance. This research was supported by the USDA Tropical/Subtropical Agriculture Research (T-STAR) Program. soilless mixes amended with up to $50 \%$ compost (biosolids and yard trimmings) without adversely affecting size, appearance, or flowering. Plants grown in media with higher percentages of compost $(75 \%$ or $100 \%)$ were still considered marketable, but were reduced in size and appeared to have abnormal root distribution (Wilson et al., 2001). Visual inspection of roots revealed an abnormal distribution along the sides of the containers with minimal root growth in the center. Manual watering may not be the optimal method of irrigating plants grown in media with high amounts of compost. Drip irrigation and subirrigation systems are commonly used in containerized perennial crop production. Dole et al. (1994) reported that microtube irrigation allowed more water to be retained in the medium and produced plants with a larger dry mass than capillary mats, ebb and flow, or manual watering. Adoption of subirrigation technology has recently increased, particularly for large-scale nurseries (Uva et al., 1998). Using subirrigation in greenhouse crop production can reduce water, fertilizer, runoff, and labor, while improving crop appearance and uniformity (Uva et al., 1998). However, the effectiveness of subirrigation depends on the substrate and irrigation frequency (D'Angelo et al., 1995). Therefore, our research objectives were to a) evaluate three irrigation methods for production of three containerized perennial salvia species grown in high percentages of organic-based media; b) characterize physical and chemical characteristics and nutrient compositions of mixtures of compost as viable potting media alternatives to a traditional, commercially available peat-based potting medium; and c) evaluate plant growth, development, nutritional status, and consumer quality of three salvia species as influenced by organic-based 
media composition and irrigation systems. Three perennial species of salvia were chosen for this study due to their increasing popularity among consumers (Clebsch, 2001) and proven performance in Florida (staff writer, Ornamental Outlook, 2001).

\section{Materials and Methods}

Plant material and media composition. Plugs (4 weeks old from cuttings) of wine sage (Salvia 'Van Houttei') $(\approx 10.4 \mathrm{~cm}$ tall), blue anise sage $(S$. gauranitica $)(\approx 7.8 \mathrm{~cm}$ tall $)$, and indigo spires sage (Salvia 'Indigo Spires') $(\approx 11.6 \mathrm{~cm}$ tall) (Robrick Nursery, Hawthorne, Fla.) were transplanted on 17 Oct. 2000 into 3.7-L cylindrical, plastic pots filled with biosolid-yard trimmings compost amended with $50 \%$ (by vol) 1:1 commercial soilless mix (Fafard mix \#2, Apopka, Fla.). Additional containers were filled with $100 \%$ compost or $100 \%$ commercial soilless mix (70\% peat, $20 \%$ perlite, and $10 \%$ vermiculite) (Fafard mix \#2, Apopka, Fla.). Compost was generated by the Solid Waste Authority of Palm Beach County, Fla. using a 1:1 ratio (by weight) of biosolids and yard trimmings (screened to $1.3 \mathrm{~cm}$ ). Materials were composted for $18 \mathrm{~d}$ in an agitated bed system, stockpiled, and then rescreened to $1.3 \mathrm{~cm}$. Compost and peatbased media derived from the identical source were used for each experiment and subsequent measurements of physical and chemical properties. A controlled-release fertilizer (15N-3.9P-10K) (Osmocote Plus; Scotts Co., Marysville, Ohio) was incorporated into all media at a rate of $6.1 \mathrm{~g} \cdot \mathrm{L}^{-1}$. All plants were treated with a $1 \%$ granular systemic insecticide imidacloprid (1-[(6-chloro-3pyridinyl)methyl]-N-nitro-2-imidazolidinimine; Olympic Horticultural Products, Bradenton, Fla.) at a standard rate of $0.37 \mathrm{~g} \cdot \mathrm{L}^{-1}$ and a broad spectrum systemic fungicide etridiazole (5-ethoxy-3trichloromethyl -1,2,4-thiadiazole), thiophanate-methyl (Dimethyl [1,2-phenylene) bis (imminocarbonothioyl) bis [carbamate]; Scotts Co.) at a standard rate of $12.9 \mathrm{~g} \cdot \mathrm{L}^{-1}$. Mean minimum and maximum temperatures in the greenhouse were 17.2 and 35.2 ${ }^{\circ} \mathrm{C}$ during the experiment.

Physical and elemental properties of media. Five replications of each medium $(0 \%, 50 \%$, and $100 \%$ compost) were evaluated initially for moisture percent, air-filled porosity (AFP), total porosity (TP), bulk density (BD), and particle density (PD). Percent moisture was determined by oven-drying water-saturated media at $105^{\circ} \mathrm{C}$ for $24 \mathrm{~h}$ and weighing before and after. The AFP was determined in $500 \mathrm{~mL}$ containers using the Wolverhampton submersion method of measuring the volume of drainage water in relation to the substrate volume (Bragg and Chambers, 1988). Standard drying procedures were then used after volume displacement methods to determine TP, BD, and PD (see Niedziela and Nelson, 1992, for equations).

Four samples from each medium $(0 \%, 50 \%$, and $100 \%$ compost) were collected (prior to adding slow release fertilizer) to determine chemical and nutrient composition. A 1 media : 2 deionized $\mathrm{H}_{2} \mathrm{O}$ extract was prepared for each mixture. Electrical conductivity (EC) was measured with a conductance meter (model 35; Yellow Springs Instrument, Yellow Springs, Ohio) and $\mathrm{pH}$ was measured with a pH meter (model 520A; Orion Research Inc., Boston, Mass.).

Media samples $(0 \%, 50 \%$ and $100 \%$ compost) were ovendried for $2 \mathrm{~d}$ at $60{ }^{\circ} \mathrm{C}$ and ground to a powder with a ball mill prior to combustion (Nelson and Sommers, 1996). Total C and N concentrations were determined by a CNS analyzer (Carlo-Erba Na-1500; BICO, Burbank, Calif.). Compost extracts (25 g; dry weight equivalent compost mix with distilled water) were pre- pared and allowed to equilibrate prior to centrifuging $\left(5,000 g_{\mathrm{n}}\right)$ for $15 \mathrm{~min}$. The supernatant was collected, filtered through Whatman filter paper (no. 42), and analyzed for $\mathrm{NO}_{3}-\mathrm{N}$ and $\mathrm{NH}_{4}$ N colorimetrically with an autoanalyzer (Alpkem; OI Analytical, Wilsonville, Ore). The Environmental Protection Agency (EPA) method 3050 (USEPA, 1998) was used to determine total P, K, $\mathrm{Ca}, \mathrm{Mg}, \mathrm{Fe}, \mathrm{Zn}, \mathrm{Cu}, \mathrm{Mn}, \mathrm{B}, \mathrm{Mo}, \mathrm{Cd} ; \mathrm{Pb}, \mathrm{Ni}$, and Al. An acid digestion procedure was used to prepare the samples for analysis by inductively coupled plasma argon spectroscopy (ICPAS) (model 61E, Thermo Jarrell Ash Corp, Franklin, Mass.). Samples were air-dried for $2 \mathrm{~d}$ and ground to a powder with a ball mill grinder. A portion of the sample $(1.0 \mathrm{~g})$ was digested in nitric acid then treated with $30 \%$ hydrogen peroxide. The sample was then refluxed with nitric acid, filtered through Whatman filter paper (no. 41), and diluted to $100 \mathrm{~mL}$ for analyses.

Irrigation TREATMENTS. Plants of each species were randomly placed in one of three irrigation treatments: manual watering, drip irrigation, or ebb and flow. Manual irrigation consisted of watering plants three times a week thoroughly until drainage occurred. Drip irrigation consisted of installing medium flow emitters (Roberts Irrigation Products, Inc., San Marcos, Calif.) in each pot and connecting them to an automatic timer set to irrigate plants $4 \mathrm{~d}$ a week for a 1 -min period $(\approx 450 \mathrm{~mL}$ water). This volume was chosen to allow leaching from each medium to occur after each irrigation. A JetFlow (American Agritech, Tempe, Ariz.) modular system was used for ebb-and-flow irrigation and set to subirrigate plants $4 \mathrm{~d}$ a week for 30 min during the first 4 weeks, and $7 \mathrm{~d}$ a week for $30 \mathrm{~min}$ for the last 2 weeks. To minimize algae growth, $0.62 \mathrm{~g} \cdot \mathrm{L}^{-1}$ sodium hypochlorite was added to the ebb-and-flow water reserve tank as described by Chase and Conover (1993). Volumetric water content of each container was determined using a reflectometer (HydroSense; Spectrum Technologies, Inc. Plainfield, Ill.) equipped with a $12-\mathrm{cm}$ probe that was inserted into the soil once every 2 weeks, $24 \mathrm{~h}$ after plants were irrigated.

Plant growth, DeVelopment, and nutritional status. Stem length, leaf greenness, flower number, visual quality, and leaf and stem dry weights were measured after 6 weeks. Stem length was measured from the soil level to the shoot apex of the primary stem. Leaf greenness was measured on the $4^{\text {th }}, 5^{\text {th }}$, and $6^{\text {th }}$ leaf from the apex of each plant using a chlorophyll meter (SPAD-502; Spectrum Technologies Inc., Plainfield, Ill.). Flowering was recorded as the number of flower spikes per plant. Visual quality ratings consisted of visual assessments by three individuals on a scale of 1 to 5 using the following parameters: 1 = very poor quality, severe leaf necrosis, leaf yellowing, no flowers, poor growth, not marketable; 2 = poor quality, large areas of leaf necrosis, leaf yellowing, few flowers, reduced growth, not marketable; 3 = fair quality, marginal plant and flower development, marginally marketable; 4 = good quality, no yellowing, good plant form and flower development, marketable; and 5 = excellent quality, no leaf necrosis, no yellowing, excellent plant form and flower development, highly marketable.

Dry weight of plants was determined by severing shoots at the crown and separately drying leaves and stems for 1 week at $70^{\circ} \mathrm{C}$. To determine the nutritional status of plants, leaves were further dried for $48 \mathrm{~h}$ at $85^{\circ} \mathrm{C}$ and ground through a Wiley mill (A.H. Thomas, Co., Philadelphia, Pa.) and passed through a $0.4-\mathrm{mm}$ sieve. Ground tissue $(0.5 \mathrm{~g})$ was digested in a solution of $5 \mathrm{~mL}$ $\mathrm{HNO}_{3}$ and $2 \mathrm{~mL}$ hydrogen peroxide using a microwave digestion system (O.I. Analytical, College Station, Texas). The concentrations of $\mathrm{P}, \mathrm{K}, \mathrm{Ca}, \mathrm{Mg}, \mathrm{Zn}, \mathrm{Cu}, \mathrm{Mn}, \mathrm{Fe}, \mathrm{B}$, and $\mathrm{Al}$ in the digest were 
Table 1. Physical properties of compost- and peat-based media. ${ }^{\mathrm{z}}$

\begin{tabular}{|c|c|c|c|c|c|c|c|}
\hline Medium $^{\mathrm{y}}$ & $\begin{array}{c}\text { Initial } \\
\text { moisture } \\
(\%)\end{array}$ & $\mathrm{pH}$ & $\begin{array}{c}\mathrm{EC} \\
\left(\mathrm{mmho} \cdot \mathrm{cm}^{-1}\right)\end{array}$ & $\begin{array}{l}\text { Air-filled } \\
\text { porosity } \\
\text { (\% by vol) }\end{array}$ & $\begin{array}{c}\text { Total } \\
\text { porosity } \\
(\% \text { by vol })\end{array}$ & $\begin{array}{c}\text { Bulk } \\
\text { density } \\
\left(\mathrm{g} \cdot \mathrm{cm}^{-3}\right)\end{array}$ & $\begin{array}{l}\text { Particle } \\
\text { density } \\
\left(\mathrm{g} \cdot \mathrm{cm}^{-3}\right)\end{array}$ \\
\hline$\overline{\text { Compost (CT) }}$ & 29.5 & 7.04 & 7.58 & 8.39 & 85.9 & 0.27 & 0.66 \\
\hline CT (50\%): peat (PT) $(50 \%)$ & 63.3 & 6.79 & 4.99 & 7.74 & 80.4 & 0.17 & 0.47 \\
\hline PT & 78.7 & 6.47 & 1.21 & 7.96 & 77.0 & 0.09 & 0.28 \\
\hline $\operatorname{LSD}_{(0.05)}$ & 21.3 & 0.25 & 0.52 & NS & 3.67 & 0.01 & 0.06 \\
\hline
\end{tabular}

${ }^{\mathrm{z}}$ Data measured before transplanting.

${ }^{\mathrm{y}} \mathrm{CT}=$ compost $(50 \%$ yard waste $: 50 \%$ biosolids $)$; $\mathrm{PT}=$ commercial peat-based mix.

determined using an inductively coupled plasma argon emission spectrometer (JY 46 P; Jobin Yvon Horiba, Edison, N.J.). Three subsamples were obtained from every replicate for each element. Total $\mathrm{N}$ concentrations in tissue samples were determined using a C-N-S analyzer (Carlo-Erba NA-1500; BICO, Burbank, Calif.) using the procedure described by Bremner (1996).

EXPERIMENTAL DESIGN AND STATISTICAL ANALYSIS. For each plant species, a split-block experimental design was used with the main blocks as irrigation methods and the split plots as media. Each medium was replicated four times. An analysis of variance (ANOVA) was performed on each collected variable using the SAS statistical software program (SAS Institute, Inc., 1989). Media $\times$ irrigation system $(M \times I S)$ interaction means are presented for variables with significant $\mathrm{M} \times \mathrm{IS}$, otherwise, main effect means of media and irrigation are presented. Variables with significant $\mathrm{M} \times \mathrm{IS}$ interactions or main effect (media or irrigation) means were separated by a LSD test, $P<0.05$.

\section{Results}

Physical, Chemical, and nutrient characteristics of the MEDIA. Media $\mathrm{pH}$ ranged from 6.47 (peat-based mix) to 6.79 and 7.04 in the $50 \%$ and $100 \%$ compost, respectively (Table 1). The $\mathrm{pH}$ of composts can range from 7.0 for composted hardwood bark (Gartner et al., 1974), 6.5 to 8.0 for municipal solid waste (MSW) (Zucconi and de Bertoldi, 1991), and 7.0 to 7.1 for biosolid:yardwaste compost (Wilson et al., 2001, 2002). In our study, media amended with compost were within an acceptable range ( $\mathrm{pH} 6.7$ to 7.7 ) for commercially produced compost (Fitzpatrick et al.,1998). However, Joiner (1981) suggested that media $\mathrm{pH}$ value for container-grown foliage plants should range between 5.5 to 6.5 .
Electrical conductivities (EC) were elevated when compost was mixed in the media (Table 1). Media with a high salt content can have deleterious effects on subsequent plant growth and development (Conover and Joiner, 1966; Lumis and Johnson, 1982; Sanderson, 1980), particularly to salt-sensitive species. Poole et al. (1981) recommended that EC values range between 0.63 to $1.56 \mathrm{dS} \cdot \mathrm{m}^{-1}$ for media used for container-grown foliage plants. Bernstein (1975) reported that EC values $>3.5 \mathrm{dS} \cdot \mathrm{m}^{-1}$ can have adverse effects on seed germination and seedling growth. Media amended with 50\% compost or compost alone had EC values above those recommended (Table 1). High EC values were also reported for other biosolids:yard waste composts (Sanderson, 1980; Shiralipour et al., 1996; Vavrina, 1994).

Initial moisture content was significantly higher for the peatbased medium than for the $50 \%$ or $100 \%$ compost media (Table 1). Air filled porosity did not differ among media (Table 1). Wootton et al. (1981) reported that as particle size of compostdigested medium increased, air filled porosity increased but plant shoot dry weight decreased. Compost $(50 \%$ or $100 \%)$ had higher particle density and bulk density than the peat-based medium (Table 1). Similar results with a biosolid:yard waste compost amended in a peat or coir-based medium were reported by Wilson et al. (2001). This suggests that although the higher density (bulk and particle) of the compost was increasing total porosity, it may simultaneously be reducing water retention of the medium.

The $\mathrm{C}$ and $\mathrm{N}$ contents were higher in media with $50 \%$ or $100 \%$ compost than the peat-based medium (Table 2). Organic wastes have been reported as a valuable source of N (Sims, 1995). This resulted in C:N ratios of 14.5 and 12.5 for the media amended with $50 \%$ or $100 \%$ compost, respectively. Composts with C:N ratios $<20$ are considered stable and optimum for plant growth (Davidson et al., 1994), while those with ratios greater than 30 may result in

Table 2. Nutrient characteristics of compost- and peat-based media. ${ }^{\mathrm{z}}$

\begin{tabular}{|c|c|c|c|c|c|c|c|c|}
\hline \multirow[b]{2}{*}{ Medium $^{y}$} & \multirow{2}{*}{$\begin{array}{l}\mathrm{C} / \mathrm{N} \\
\text { ratio }\end{array}$} & \multicolumn{6}{|c|}{ Concn $\left(\mathrm{g} \cdot \mathrm{kg}^{-1}\right)$} & \\
\hline & & $\mathrm{N}$ & $\mathrm{C}$ & $\mathrm{P}$ & $\mathrm{K}$ & $\mathrm{Ca}$ & $\mathrm{Mg}$ & \\
\hline$\overline{\text { Compost (CT) }}$ & 12.2 & 25.0 & 304 & 10.8 & 6.9 & 47.7 & 3.0 & \\
\hline CT (50\%): peat (PT) $(50 \%)$ & 14.5 & 20.0 & 291 & 9.4 & 7.3 & 38.4 & 6.9 & \\
\hline PT & 46.8 & 5.8 & 271 & 0.4 & 6.8 & 15.8 & 22.0 & \\
\hline \multirow[t]{2}{*}{$\operatorname{LSD}_{(0.05)}$} & 0.6 & 1.20 & 150 & 0.7 & 0.3 & 9.3 & 7.4 & \\
\hline & \multicolumn{8}{|c|}{ Concn $\left(\mathrm{mg} \cdot \mathrm{kg}^{-1}\right)$} \\
\hline Medium $^{y}$ & $\mathrm{Zn}$ & $\mathrm{Cu}$ & $\mathrm{Mn}$ & $\mathrm{Al}$ & $\mathrm{Fe}$ & B & $\mathrm{Cd}$ & $\mathrm{Pb}$ \\
\hline$\overline{\mathrm{CT}}$ & 297 & 187 & 154 & 3650 & 16325 & 21.3 & 1.25 & 28.5 \\
\hline CT (50\%): PT (50\%) & 230 & 170 & 127 & 4955 & 23375 & 15.3 & 1.75 & 20.3 \\
\hline PT & 36 & 22 & 150 & 8950 & 14800 & 7.0 & 1.00 & 2.8 \\
\hline $\operatorname{LSD}_{(0.05)}$ & 23 & 11 & 11 & 239 & 948 & 1.8 & 0.64 & 6.9 \\
\hline
\end{tabular}

${ }^{\mathrm{z}}$ Data measured before transplanting.

${ }^{\mathrm{y}} \mathrm{CT}=$ compost $(50 \%$ yard waste $: 50 \%$ biosolids $) ; \mathrm{PT}=$ commercial peat-based mix. 
Table 3. Media and irrigation effects on plant growth and quality of Salvia 'Van Houttei'.

\begin{tabular}{|c|c|c|c|c|c|c|c|}
\hline $\operatorname{Medium}(\mathrm{M})^{\mathrm{y}}$ & $\begin{array}{l}\text { Irrigation } \\
\text { system } \\
\text { (IS) }\end{array}$ & $\begin{array}{c}\text { Stem } \\
\text { length } \\
(\mathrm{cm})\end{array}$ & $\begin{array}{c}\text { Leaf } \\
\text { greenness } \\
\text { (SPAD } \\
\text { units) }\end{array}$ & $\begin{array}{c}\text { Flower } \\
\text { spikes } \\
\text { (no.) }\end{array}$ & $\begin{array}{l}\text { Leaf } \\
\text { dry wt } \\
\text { (g) }\end{array}$ & $\begin{array}{c}\text { Stem } \\
\text { dry wt } \\
(\mathrm{g})\end{array}$ & $\begin{array}{l}\text { Visual } \\
\text { quality } \\
\text { rating }^{\mathrm{x}}\end{array}$ \\
\hline$\overline{\text { Compost (CT) }}$ & & 67.8 & 35.7 & & 5.25 & 4.42 & \\
\hline $\mathrm{CT}(50 \%)$ : peat (PT) $(50 \%)$ & & 63.7 & 32.9 & & 4.72 & 3.53 & \\
\hline PT & & 73.8 & 37.0 & & 5.16 & 5.14 & \\
\hline $\operatorname{LSD}_{(0.05)}$ & & 6.8 & 2.2 & & & 0.98 & \\
\hline & Ebb flow & 71.6 & 33.6 & & 6.34 & 5.43 & \\
\hline & Drip & 70.1 & 36.0 & & 3.85 & 3.67 & \\
\hline & Manual & 63.7 & 36.0 & & 4.93 & 3.99 & \\
\hline $\operatorname{LSD}_{(0.05)}$ & & 5.4 & & & 0.65 & 1.17 & \\
\hline $\mathrm{CT}$ & Ebb flow & & & 11.3 & & & 3.33 \\
\hline $\mathrm{CT}$ & Drip & & & 8.8 & & & 3.00 \\
\hline $\mathrm{CT}$ & Manual & & & 10.8 & & & 5.00 \\
\hline CT $(50 \%)$ : PT (50\%) & Ebb flow & & & 15.0 & & & 3.67 \\
\hline CT $(50 \%)$ : PT (50\%) & Drip & & & 6.5 & & & 2.00 \\
\hline $\mathrm{CT}(50 \%)$ : PT (50\%) & Manual & & & 12.5 & & & 3.00 \\
\hline PT & Ebb flow & & & 15.8 & & & 4.33 \\
\hline PT & Drip & & & 15.5 & & & 4.33 \\
\hline PT & Manual & & & 11.5 & & & 4.00 \\
\hline $\begin{array}{l}\mathrm{LSD}_{(0.05)}(\mathrm{M} \times \mathrm{IS}) \\
\text { Significance }\end{array}$ & & & & 4.6 & & & 0.77 \\
\hline M & & $*$ & $* *$ & $*$ & NS & $*$ & $* *$ \\
\hline IS & & $*$ & NS & NS & $* *$ & $*$ & $* *$ \\
\hline $\mathrm{M} \times \mathrm{IS}$ & & NS & NS & $*$ & NS & NS & $* *$ \\
\hline
\end{tabular}

${ }^{\mathrm{z}}$ Data measured 6 weeks after transplanting.

${ }^{\mathrm{y}} \mathrm{CT}=$ compost $(50 \%$ yard waste $: 50 \%$ biosolids); PT $=$ commercial peat-based mix.

${ }^{\mathrm{x}}$ Visual quality rating: 1 = very poor quality, not marketable; 2 = poor quality, not marketable; 3 = fair quality, marginally marketable; 4 = good quality, marketable; 5 = excellent quality, highly marketable.

Ns,*,**Nonsignificant, or significant $\mathrm{F}$ test at $P \leq 0.05$, or 0.01 , respectively.

plant phytotoxicity and $\mathrm{N}$ immobilization (Zucconi et al., 1981).

Compost-based media (50\% or $100 \%)$ had higher $\mathrm{Ca}$ and $\mathrm{P}$ contents but lower $\mathrm{Mg}$ content than the peat-based medium (Table 2). Manganese (Mn) content was significantly lower and $\mathrm{K}$ higher in the $50 \%$ compost amended medium then that of the $100 \%$ compost or peat-based medium. Compost-based media (50\% or $100 \%$ ) had higher $\mathrm{Zn}, \mathrm{Cu}, \mathrm{Fe}$, and B contents but lower $\mathrm{Al}$ contents than the peat-based medium (Table 2). Although the compost-based medium had higher $\mathrm{Pb}$ content than the peatbased medium (Table 2), heavy metal ( $\mathrm{Pb}$ or $\mathrm{Cd}$ ) contents did not exceed the Environmental Protection Agency (EPA) levels for biosolids application (USEPA, 1994) for any substrate.

MEDIA AND IRRIGATION INFLUENCE ON 'VAN HOUTTEI': EXPT. 1. Media $\times$ irrigation interactions occurred only for flower spike number and visual quality ratings (Table 3 ). Plants grown in $100 \%$ compost had similar stem lengths, leaf dry weights, and stem dry weights as plants grown in peat-based media. Plants grown in 50\% compost had significantly reduced heights (stem lengths) and stem dry weights than did plants grown in peat-based media.

Plants grown using ebb-and-flow irrigation system were taller than manually watered plants and had higher leaf and stem dry weights than the drip irrigation or manual watering systems (Table 3). Plants had a similar number of flower spikes among irrigation systems for plants grown in $100 \%$ compost or the peatbased medium. Plants grown in 50\% compost and irrigated using ebb-and-flow or manual systems had more flower spikes than plants grown using drip irrigation.

SPAD leaf readings, an indicator of leaf greenness, did not differ among irrigation systems (Table 3 ). Overall visual quality ratings were highest (improved appearance) for plants grown in the peat-based media than in compost-based $(50 \%$ or $100 \%)$ media irrigated by drip (Table 3). However, plants grown in $100 \%$ compost had a higher visual quality rating than plants grown in 50\% compost with manual watering. Regardless, all media or irrigation systems resulted in plants that were of marketable quality, except for plants grown in 50\% compost using drip irrigation (Table 3).

Leaf $\mathrm{N}$ concentration was higher in plants grown in 50\% compost media than in $100 \%$ compost or peat-based media (Table 4). Media did not influence leaf $\mathrm{Ca}, \mathrm{Cu}, \mathrm{Fe}$ or $\mathrm{Al}$ contents (Table 4). Plants grown in $100 \%$ compost had higher leaf $\mathrm{K}$ and $\mathrm{B}$, but lower $\mathrm{Mg}$ contents, than plants grown in the peat-based medium. Irrigation systems did not influence leaf $\mathrm{N}, \mathrm{Ca}, \mathrm{Mg}, \mathrm{Cu}, \mathrm{Fe}$, and $\mathrm{B}$ contents (Table 4). However, plants irrigated using ebb and flow had higher leaf $\mathrm{K}$ content than manual or drip irrigation systems. Leaf Mn content was higher in plants irrigated manually than ebb-and-flow or drip systems. Leaf Al content was higher in plants grown using drip irrigation than ebb-and-flow or manual systems. Media $\times$ irrigation interaction was significant for leaf $P$ content. However, leaf $\mathrm{P}$ content was higher when plants were grown in $100 \%$ compost than in the $100 \%$ peat-based medium, regardless of irrigation system (Table 4), which may be attributed to the higher initial $\mathrm{P}$ concentration in the $100 \%$ compost (Table 2). The significant media $\times$ irrigation interaction for leaf $\mathrm{Zn}$ content was primarily attributed to the higher leaf $\mathrm{Zn}$ content in plants grown in $100 \%$ compost and irrigated by ebb and flow compared with the other treatments. 
Table 4. Elemental contents of Salvia 'Van Houttei' grown for 6 weeks in compost or peat-based media.

\begin{tabular}{|c|c|c|c|c|c|c|c|c|c|c|c|c|}
\hline \multirow[b]{2}{*}{$\operatorname{Medium}(\mathrm{M})^{\mathrm{z}}$} & \multirow{2}{*}{$\begin{array}{l}\text { Irrigation } \\
\text { system } \\
\text { (IS) }\end{array}$} & \multicolumn{5}{|c|}{ Concn $\left(\mathrm{g} \cdot \mathrm{kg}^{-1}\right)$} & \multicolumn{6}{|c|}{ Concn $\left(\mathrm{mg} \cdot \mathrm{kg}^{-1}\right)$} \\
\hline & & $\mathrm{N}$ & $\mathrm{P}$ & $\mathrm{K}$ & $\mathrm{Ca}$ & $\mathrm{Mg}$ & $\mathrm{Zn}$ & $\mathrm{Cu}$ & $\mathrm{Mn}$ & $\mathrm{Fe}$ & $\mathrm{B}$ & $\mathrm{Al}$ \\
\hline$\overline{\text { Compost }(\mathrm{CT})}$ & & 51.9 & & 37.7 & 13.5 & 4.7 & & 18.7 & 39.8 & 125 & 60.5 & 106 \\
\hline $\mathrm{CT}(50 \%)$ : peat (PT) $(50 \%)$ & & 57.4 & & 36.6 & 13.1 & 5.3 & & 18.7 & 18.4 & 109 & 41.6 & 108 \\
\hline PT & & 52.0 & & 27.2 & 13.1 & 6.6 & & 16.6 & 35.3 & 113 & 35.7 & 109 \\
\hline \multirow[t]{4}{*}{$\operatorname{LSD}_{(0.05)}$} & & 4.8 & & 3.8 & & 0.7 & & & 5.0 & & 13.8 & \\
\hline & Ebb flow & 56.5 & & 43.3 & 13.0 & 5.5 & & 18.4 & 24.5 & 109 & 46.4 & 101 \\
\hline & Drip & 53.8 & & 29.0 & 13.5 & 5.3 & & 17.5 & 27.0 & 118 & 45.9 & 116 \\
\hline & Manual & 51.0 & & 29.4 & 13.3 & 5.8 & & 18.2 & 33.0 & 120 & 45.5 & 106 \\
\hline $\operatorname{LSD}_{(0.05)}$ & & & & 6.2 & & & & & 5.4 & & & 6 \\
\hline CT & Ebb flow & & 68.4 & & & & 104 & & & & & \\
\hline CT & Drip & & 81.0 & & & & 81 & & & & & \\
\hline CT & Manual & & 77.2 & & & & 79 & & & & & \\
\hline CT (50\%) : PT (50\%) & Ebb flow & & 66.2 & & & & 75 & & & & & \\
\hline CT $(50 \%):$ PT $(50 \%)$ & Drip & & 76.5 & & & & 71 & & & & & \\
\hline CT $(50 \%):$ PT (50\%) & Manual & & 67.5 & & & & 63 & & & & & \\
\hline PT & Ebb flow & & 53.8 & & & & 67 & & & & & \\
\hline PT & Drip & & 36.1 & & & & 74 & & & & & \\
\hline PT & Manual & & 49.2 & & & & 78 & & & & & \\
\hline $\mathrm{LSD}_{(0.05)}(\mathrm{M} \times \mathrm{IS})$ & & & 14.1 & & & & 17 & & & & & \\
\hline \multicolumn{13}{|l|}{ Significance } \\
\hline M & & $*$ & $* *$ & $* *$ & NS & $* *$ & $* *$ & NS & $* *$ & NS & $* *$ & NS \\
\hline IS & & NS & NS & $* *$ & NS & NS & NS & NS & $*$ & NS & NS & $* *$ \\
\hline $\mathrm{M} \times \mathrm{IS}$ & & NS & $*$ & NS & NS & NS & $*$ & NS & NS & NS & NS & NS \\
\hline
\end{tabular}

${ }^{\mathrm{z}} \mathrm{CT}=$ compost $(50 \%$ yard waste $: 50 \%$ biosolids); $\mathrm{PT}=$ commercial peat-based mix.

Ns, ${ }^{*}{ }^{* *}$ Nonsignificant, or significant $\mathrm{F}$ test at $P \leq 0.05$, or 0.01 , respectively.

Table 5. Media and irrigation effects on plant growth and quality of Salvia gauranitica 'Black and Blue'.

\begin{tabular}{|c|c|c|c|c|c|c|c|}
\hline $\operatorname{Medium}(\mathrm{M})^{\mathrm{y}}$ & $\begin{array}{l}\text { Irrigation } \\
\text { system } \\
\text { (IS) }\end{array}$ & $\begin{array}{l}\text { Stem } \\
\text { length } \\
(\mathrm{cm})\end{array}$ & $\begin{array}{c}\text { Leaf } \\
\text { greenness } \\
\text { (SPAD } \\
\text { units) }\end{array}$ & $\begin{array}{c}\text { Flower } \\
\text { spikes } \\
\text { (no.) }\end{array}$ & $\begin{array}{l}\text { Leaf } \\
\text { dry wt } \\
(\mathrm{g})\end{array}$ & $\begin{array}{l}\text { Stem } \\
\text { dry wt } \\
(\mathrm{g})\end{array}$ & $\begin{array}{l}\text { Visual } \\
\text { quality } \\
\text { rating }\end{array}$ \\
\hline$\overline{\text { Compost (CT) }}$ & & & & & & 2.19 & 3.67 \\
\hline CT $(50 \%)$ : peat (PT) $(50 \%)$ & & & & & & 2.76 & 3.00 \\
\hline PT & & & & & & 2.80 & 4.44 \\
\hline \multirow{4}{*}{$\operatorname{LSD}_{(0.05)}$} & & & & & & & 0.50 \\
\hline & Ebb flow & & & & & 2.75 & 3.33 \\
\hline & Drip & & & & & 2.86 & 3.89 \\
\hline & Manual & & & & & 2.15 & 3.89 \\
\hline \multicolumn{8}{|l|}{$\operatorname{LSD}_{(0.05)}$} \\
\hline $\mathrm{CT}^{(0.05)}$ & Ebb flow & 33.5 & 33.5 & 1.75 & 3.35 & & \\
\hline $\mathrm{CT}$ & Drip & 47.9 & 32.6 & 6.25 & 5.61 & & \\
\hline $\mathrm{CT}$ & Manual & 45.2 & 32.0 & 3.00 & 4.96 & & \\
\hline CT (50\%): PT (50\%) & Ebb flow & 46.2 & 27.0 & 5.25 & 5.83 & & \\
\hline CT (50\%): PT (50\%) & Drip & 44.0 & 32.7 & 2.75 & 4.06 & & \\
\hline CT (50\%): PT (50\%) & Manual & 39.0 & 32.5 & 2.50 & 4.90 & & \\
\hline $\mathrm{PT}$ & Ebb flow & 54.4 & 33.0 & 5.50 & 7.29 & & \\
\hline PT & Drip & 50.5 & 33.1 & 3.25 & 5.87 & & \\
\hline PT & Manual & 47.1 & 34.6 & 3.00 & 5.85 & & \\
\hline $\mathrm{LSD}_{(0.05)}(\mathrm{M} \times \mathrm{IS})$ & & 8.4 & 2.0 & 3.03 & 1.30 & & \\
\hline \multicolumn{8}{|l|}{ Significance } \\
\hline M & & $* *$ & $* *$ & NS & $* *$ & NS & $* *$ \\
\hline IS & & NS & NS & NS & NS & NS & NS \\
\hline $\mathrm{M} \times \mathrm{IS}$ & & $* *$ & $* *$ & $*$ & $* *$ & NS & NS \\
\hline
\end{tabular}

${ }^{\mathrm{z}}$ Data measured 6 weeks after transplanting.

${ }^{\mathrm{y}} \mathrm{CT}=$ compost $(50 \%$ yard waste $: 50 \%$ biosolids); $\mathrm{PT}=$ commercial peat-based mix.

${ }^{\mathrm{x}}$ Visual quality rating: 1 = very poor quality, not marketable; 2 = poor quality, not marketable; 3 = fair quality, marginally marketable; 4 = good quality, marketable; 5 = excellent quality, highly marketable.

NS,*,** Nonsignificant, or significant $\mathrm{F}$ test at $P \leq 0.05$, or 0.01 , respectively. 
MEDIA AND IRRIGATION INFLUENCE ON $S$. GAURANITICA: EXPT. 2. Media $\times$ irrigation interactions were significant for stem length, leaf greenness, number of flower spikes, and leaf dry weight (Table 5). Stem lengths were similar between irrigation systems when plants were grown in peat-based or $50 \%$ compost media, but plants were taller when grown in $100 \%$ compost with drip or manual irrigations compared to ebb-and-flow irrigation (Table 5). Leaf greenness measurements were similar among irrigation systems for plants grown in $100 \%$ compost or peat-based media.
However, plants grown in 50\% compost and irrigated by ebband-flow system had reduced leaf greenness compared to drip or manual irrigation. Number of flower spikes was similar from plants grown in peat-based media or 50\% compost regardless of irrigation system but plants grown in 100\% compost had more flowers spikes using drip irrigation than ebb-and-flow or manual irrigation systems. Plants grown in the peat-based media or $100 \%$ compost had leaf dry weights higher or lower, respectively, using the ebb-and-flow irrigation system than the other irrigation sys-

Table 6. Elemental contents of Salvia gauranitica 'Black and Blue' grown for 6 weeks in compost or peat-based media.

\begin{tabular}{|c|c|c|c|c|c|c|c|c|c|c|c|c|}
\hline \multirow[b]{2}{*}{$\operatorname{Medium}(\mathrm{M})^{\mathrm{z}}$} & \multirow{2}{*}{$\begin{array}{l}\text { Irrigation } \\
\text { system } \\
\text { (IS) }\end{array}$} & \multicolumn{5}{|c|}{ Concn $\left(\mathrm{g} \cdot \mathrm{kg}^{-1}\right)$} & \multicolumn{6}{|c|}{ Concn $\left(\mathrm{mg} \cdot \mathrm{kg}^{-1}\right)$} \\
\hline & & $\mathrm{N}$ & $\mathrm{P}$ & $\mathrm{K}$ & $\mathrm{Ca}$ & $\mathrm{Mg}$ & $\mathrm{Zn}$ & $\mathrm{Cu}$ & $\mathrm{Mn}$ & $\mathrm{Fe}$ & $\mathrm{B}$ & $\mathrm{Al}$ \\
\hline$\overline{\text { Compost (CT) }}$ & & 43.9 & & 53.1 & 12.3 & 7.1 & 65.5 & 21.6 & & 93 & & 81.5 \\
\hline CT (50\%): peat (PT) $(50 \%)$ & & 48.7 & & 52.9 & 12.2 & 8.0 & 50.7 & 21.6 & & 98 & & 83.9 \\
\hline PT & & 50.0 & & 47.5 & 12.2 & 10.3 & 61.4 & 17.8 & & 132 & & 94.3 \\
\hline \multirow[t]{4}{*}{$\operatorname{LSD}_{(0.05)}$} & & 3.0 & & 4.6 & & 0.6 & & 2.5 & & 34 & & 9.8 \\
\hline & Ebb flow & 49.3 & & 55.1 & 11.5 & 8.0 & 62.4 & 18.2 & & 97 & & 71.5 \\
\hline & Drip & 45.2 & & 49.2 & 12.8 & 8.9 & 52.4 & 21.3 & & 117 & & 98.7 \\
\hline & Manual & 48.1 & & 49.1 & 12.5 & 8.6 & 62.8 & 21.4 & & 109 & & 89.5 \\
\hline $\operatorname{LSD}_{(0.05)}$ & & & & 4.4 & 0.6 & 0.5 & & 2.5 & & & & 8.9 \\
\hline $\mathrm{CT}^{(0.0 \mathrm{~S})}$ & Ebb flow & & 4.1 & & & & & & 51.8 & & 65.1 & \\
\hline $\mathrm{CT}$ & Drip & & 4.8 & & & & & & 73.3 & & 51.6 & \\
\hline $\mathrm{CT}$ & Manual & & 5.0 & & & & & & 68.4 & & 53.4 & \\
\hline CT (50\%): PT (50\%) & Ebb flow & & 4.5 & & & & & & 30.8 & & 47.1 & \\
\hline CT (50\%): PT (50\%) & Drip & & 4.8 & & & & & & 31.1 & & 38.2 & \\
\hline CT (50\%): PT (50\%) & Manual & & 4.5 & & & & & & 19.5 & & 35.0 & \\
\hline PT & Ebb flow & & 4.2 & & & & & & 36.0 & & 35.3 & \\
\hline PT & Drip & & 3.8 & & & & & & 29.7 & & 41.6 & \\
\hline PT & Manual & & 3.9 & & & & & & 27.4 & & 37.5 & \\
\hline $\operatorname{LSD}_{(0.05)}(\mathrm{M} \times \mathrm{IS})$ & & & 0.4 & & & & & & 11.3 & & 9.2 & \\
\hline \multicolumn{13}{|l|}{ Significance } \\
\hline M & & $* *$ & $* *$ & $*$ & NS & $* *$ & NS & $* *$ & $* *$ & $*$ & $* *$ & $*$ \\
\hline IS & & NS & NS & $*$ & $* *$ & $* *$ & NS & $*$ & NS & NS & NS & $* *$ \\
\hline $\mathrm{M} \times \mathrm{IS}$ & & NS & $* *$ & NS & NS & NS & NS & NS & $* *$ & NS & $*$ & NS \\
\hline
\end{tabular}

${ }^{\mathrm{Z}} \mathrm{CT}=$ compost $(50 \%$ yard waste $: 50 \%$ biosolids); $\mathrm{PT}=$ commercial peat-based mix.

Ns,****Nonsignificant, or significant $\mathrm{F}$ test at $P \leq 0.05$, or 0.01 , respectively.

Table 7. Media and irrigation effects on plant growth and quality of Salvia 'Indigo Spires'.

\begin{tabular}{|c|c|c|c|c|c|c|c|}
\hline $\operatorname{Medium}(\mathrm{M})^{\mathrm{y}}$ & $\begin{array}{l}\text { Irrigation } \\
\text { system } \\
\text { (IS) }\end{array}$ & $\begin{array}{l}\text { Stem } \\
\text { length } \\
(\mathrm{cm})\end{array}$ & $\begin{array}{c}\text { Leaf } \\
\text { greenness } \\
\text { (SPAD } \\
\text { units) }\end{array}$ & $\begin{array}{c}\text { Flower } \\
\text { spikes } \\
\text { (no.) }\end{array}$ & $\begin{array}{l}\text { Leaf } \\
\text { dry wt } \\
(\mathrm{g})\end{array}$ & $\begin{array}{l}\text { Stem } \\
\text { dry wt } \\
(\mathrm{g})\end{array}$ & $\begin{array}{l}\text { Visual } \\
\text { quality } \\
\text { rating }^{\mathrm{x}}\end{array}$ \\
\hline$\overline{\text { Compost (CT) }}$ & & 83.6 & 34.7 & 6.67 & 7.54 & 7.98 & 4.56 \\
\hline CT (50\%): peat (PT) $(50 \%)$ & & 83.5 & 34.6 & 6.42 & 8.26 & 7.75 & 4.00 \\
\hline PT & & 79.1 & 34.9 & 8.08 & 7.19 & 7.02 & 4.11 \\
\hline & Ebb flow & 78.1 & 34.8 & 6.17 & 8.61 & 7.48 & 4.22 \\
\hline & Drip & 87.2 & 34.7 & 7.83 & 7.33 & 8.16 & 4.11 \\
\hline & Manual & 80.9 & 34.6 & 7.17 & 7.06 & 7.10 & 4.33 \\
\hline $\begin{array}{l}\operatorname{LSD}_{(0.05)} \\
\text { Significance }\end{array}$ & & 6.2 & & & & & \\
\hline M & & NS & NS & NS & NS & NS & NS \\
\hline IS & & $*$ & NS & NS & NS & NS & NS \\
\hline $\mathrm{M} \times \mathrm{IS}$ & & NS & NS & NS & NS & NS & NS \\
\hline
\end{tabular}

${ }^{\mathrm{z}}$ Data measured 6 weeks after transplanting.

${ }^{\mathrm{y}} \mathrm{CT}=$ compost $(50 \%$ yard waste $: 50 \%$ biosolids $)$; PT $=$ commercial peat-based mix.

wVisual quality rating: 1 = very poor quality, not marketable; 2 = poor quality, not marketable; 3 = fair quality, marginally marketable; 4 = good quality, marketable; $5=$ excellent quality, highly marketable.

Ns, ${ }^{*}$ Nonsignificant, or significant $\mathrm{F}$ test at $P \leq 0.05$, respectively. 
tems. Main effects of media or irrigation system were not significant for stem dry weights (Table 5). Plants grown in the $100 \%$ peat-based medium had higher visual quality ratings than those grown in the compost-based medium (50\% or 100\%) (Table 5). Regardless, each medium had plants of acceptable consumer appearance and marketable quality. Overall visual quality ratings of $S$. gauranitica were not affected by irrigation.

Plants grown in $100 \%$ compost had lower leaf $\mathrm{N}, \mathrm{Mg}, \mathrm{Fe}$, and $\mathrm{Al}$, and higher leaf $\mathrm{K}$ and $\mathrm{Cu}$ contents than the peat-based media (Table 6). Media had no influence on leaf $\mathrm{Ca}$ and $\mathrm{Zn}$ content. Plants irrigated by ebb-and-flow system had lower leaf $\mathrm{Ca}, \mathrm{Mg}, \mathrm{Cu}$, and $\mathrm{Al}$ but higher $\mathrm{K}$ than drip or manual irrigation systems. Leaf N, Zn, or Fe content did not differ among irrigation systems. Media $\times$ irrigation interaction was significant for leaf P content. Similar leaf P content among irrigation systems occurred regardless if plants were grown in $50 \%$ compost or $100 \%$ peat-based media, but leaf $\mathrm{P}$ content was lower in plant grown in $100 \%$ compost and irrigated by ebb and flow compared with drip or manual irrigations. Although a significant media $\times$ irrigation interaction occurred for leaf $\mathrm{Mn}$ and $\mathrm{B}$ contents, plants grown in $100 \%$ compost had a higher leaf B and Mn content than when plants were grown in peat-based media, regardless of the irrigation system.

Media ANd irrigation influence on 'Indigo SpIRES': Expt. 3. Plant growth characteristics (number of flower spikes, leaf dry weight, stem dry weight), and leaf greenness were not influenced by media or irrigation systems (Table 7). Stem length was not influenced by media, but stems were shorter for plants irrigated by the manual or ebb-and-flow systems than for plants irrigated by the drip system. Visual quality ratings did not differ among irrigation systems or media (Table 7). Plants from each treatment had acceptable consumer appearance and were considered of marketable quality.
Plants grown in $100 \%$ compost had higher $\mathrm{P}$ and $\mathrm{Mn}$; lower $\mathrm{Zn}, \mathrm{Fe}, \mathrm{B}$, and $\mathrm{Al}$; but similar $\mathrm{N}$ leaf content than plants grown in peat-based media (Table 8). Significant media $\times$ irrigation interactions occurred for $\mathrm{K}, \mathrm{Ca}, \mathrm{Mg}$, and $\mathrm{Cu}$ leaf content (Table 8). A higher leaf $\mathrm{Cu}$ content occurred in plants grown in compost-based $(50 \%$ or $100 \%)$ media with ebb-and-flow irrigation than in plants grown under drip or manual irrigation systems. However, when plants were grown in peat-based media, $\mathrm{Cu}$ content was similar among irrigation systems. Leaf $\mathrm{Ca}$ content was unaffected by irrigation system with $100 \%$ peat-based or $50 \%$ compost media. However, with $100 \%$ compost, plants had lower leaf Ca contents when grown using ebb-and-flow irrigation than when using manual or drip irrigation. Leaf $\mathrm{K}$ contents were higher in plants grown in 50\% compost using ebb-and-flow irrigation than manual or drip irrigation. Leaf $\mathrm{K}$ contents were similar between plants grown using manual or drip irrigations, regardless of media. Although a significant media $\times$ irrigation interaction occurred for leaf $\mathrm{Mg}$ content, plants grown in the peat-based medium had a higher leaf $\mathrm{Mg}$ content than the $50 \%$ or $100 \%$ compost, regardless of irrigation system. Irrigation systems did not influence $\mathrm{Zn}, \mathrm{Mn}$, $\mathrm{Fe}$, or Al leaf content. Plants irrigated by ebb and flow had higher $\mathrm{N}$ and $\mathrm{K}$ and lower $\mathrm{B}$ content than plants that were irrigated manually.

\section{Discussion}

Generally, plants grown in the compost-based medium were similar or slightly reduced in size (stem length, stem dry weight, leaf dry weight) or flower spike number compared to plants grown in the peat-based medium (Tables 3, 5, and 7). Similar results were reported for other perennials such as mexican heather (Cuphea hyssopifolia H.B.K.) (Wilson et al., 2001), Bolivian

Table 8. Elemental contents of Salvia 'Indigo Spires' grown for 6 weeks in compost or peat-based media.

\begin{tabular}{|c|c|c|c|c|c|c|c|c|c|c|c|c|}
\hline \multirow[b]{2}{*}{$\operatorname{Medium}(\mathrm{M})^{\mathrm{z}}$} & \multirow{2}{*}{$\begin{array}{l}\text { Irrigation } \\
\text { system } \\
\text { (IS) }\end{array}$} & \multicolumn{5}{|c|}{ Concn $\left(\mathrm{g} \cdot \mathrm{kg}^{-1}\right)$} & \multicolumn{6}{|c|}{ Concn $\left(\mathrm{mg} \cdot \mathrm{kg}^{-1}\right)$} \\
\hline & & $\mathrm{N}$ & $\mathrm{P}$ & $\mathrm{K}$ & $\mathrm{Ca}$ & $\overline{\mathrm{Mg}}$ & $\mathrm{Zn}$ & $\mathrm{Cu}$ & $\mathrm{Mn}$ & $\mathrm{Fe}$ & $\mathrm{B}$ & $\mathrm{Al}$ \\
\hline$\overline{\text { Compost (CT) }}$ & & 43.5 & 6.6 & & & & 37.2 & & 71.0 & 53 & 11.7 & 41.0 \\
\hline CT (50\%): peat (PT) $(50 \%)$ & & 45.6 & 5.3 & & & & 76.4 & & 32.2 & 100 & 31.7 & 85.7 \\
\hline PT & & 47.9 & 3.7 & & & & 75.2 & & 22.0 & 97 & 32.6 & 88.4 \\
\hline \multirow{4}{*}{$\operatorname{LSD}_{(0.05)}$} & & & 0.6 & & & & 10.0 & & 6.0 & 13 & 5.3 & 16.1 \\
\hline & Ebb flow & 52.0 & 5.5 & & & & 69.0 & & 42.6 & 78.1 & 22.6 & 66.5 \\
\hline & Drip & 41.7 & 4.8 & & & & 59.9 & & 40.6 & 82.6 & 21.8 & 75.4 \\
\hline & Manual & 43.3 & 5.4 & & & & 60.0 & & 42.0 & 88.4 & 31.4 & 73.2 \\
\hline $\operatorname{LSD}_{(0.05)}$ & & 4.9 & 0.6 & & & & & & & & 5.7 & \\
\hline $\mathrm{CT}$ & Ebb flow & & & 33.0 & 9.3 & 4.0 & & 15.4 & & & & \\
\hline $\mathrm{CT}$ & Drip & & & 25.3 & 11.6 & 4.2 & & 8.05 & & & & \\
\hline $\mathrm{CT}$ & Manual & & & 28.8 & 12.1 & 4.2 & & 9.29 & & & & \\
\hline CT (50\%): PT (50\%) & Ebb flow & & & 59.0 & 10.4 & 5.5 & & 16.8 & & & & \\
\hline CT (50\%): PT (50\%) & Drip & & & 39.5 & 10.6 & 6.9 & & 13.2 & & & & \\
\hline CT (50\%): PT (50\%) & Manual & & & 43.0 & 11.1 & 6.6 & & 13.9 & & & & \\
\hline PT & Ebb flow & & & 41.4 & 12.3 & 9.7 & & 13.0 & & & & \\
\hline PT & Drip & & & 36.9 & 11.7 & 8.8 & & 12.6 & & & & \\
\hline PT & Manual & & & 36.3 & 11.6 & 9.5 & & 14.5 & & & & \\
\hline $\mathrm{LSD}_{(0.05)}(\mathrm{M} \times \mathrm{IS})$ & & & & 4.5 & 1.3 & 0.7 & & 2.6 & & & & \\
\hline \multicolumn{13}{|l|}{ Significance } \\
\hline M & & NS & $* *$ & $* *$ & $*$ & $* *$ & $* *$ & $* *$ & $* *$ & $* *$ & $* *$ & $* *$ \\
\hline IS & & $* *$ & $*$ & $* *$ & $*$ & NS & NS & $* *$ & NS & NS & $* *$ & NS \\
\hline $\mathrm{M} \times \mathrm{IS}$ & & NS & NS & $* *$ & $*$ & $* *$ & NSS & $* *$ & NS & NS & NS & NS \\
\hline
\end{tabular}

${ }^{\mathrm{z}} \mathrm{CT}=$ compost $(50 \%$ yard waste $: 50 \%$ biosolids); $\mathrm{PT}=$ commercial peat-based mix.

Ns,*,** Nonsignificant, or significant $\mathrm{F}$ test at $P \leq 0.05$, or 0.01 , respectively. 
sunset (Gloxinia sylvatica (HBK) Wiehler), Brazilian plume (Justicia carnea Lindl.) and golden globe (Lysimachia congestiflora Hemsl.) (Wilson et al., 2002).

Compost-based media had higher N, Ca, P, Zn, Cu, Fe, and B but lower Al content than peat-based media (Table 2). Composts, particularly those with biosolid feedstocks, have been reported to have substantial N content (Sims, 1995) and micronutrients ( $\mathrm{He}$ et al., 2001). However, $\mathrm{N}$ must mineralize before it is available for plant uptake.

Leaf $\mathrm{N}$ content of plants grown in compost alone was similar or slightly lower than plants grown in peat-based media. Leaf tissue of plants grown in $100 \%$ compost generally had higher $\mathrm{K}$, $\mathrm{P}$, and $\mathrm{Mn}$; lower $\mathrm{Mg}, \mathrm{Fe}$, and $\mathrm{Al}$; but similar Ca content than leaf tissue of plants grown in peat-based media. Although each treatment was initially fertilized with a slow-release fertilizer, increases in leaf $\mathrm{K}$ and $\mathrm{P}$ content of plants grown in compost $(50 \%$ or $100 \%$ ) may be attributed to higher available $\mathrm{K}$ and $\mathrm{P}$ contents in the compost.

Stem dry weights for each species did not respond differentially between media and irrigation systems. Leaf greenness was generally similar among irrigation systems. In general, plants irrigated by ebb and flow resulted in heavier ('Van Houttei) or similar ('Indigo Spires', S. gauranitica) dry stem weights than plants irrigated manually or by drip. Klock-Moore and Broschat (2001) reported that ebb and flow or overhead irrigations resulted in similar plant shoot growth for pentas [Pentas lanceolata (Forssk.) Deflers], crossandra [Crossandra infundibuliformis (L.) Nees.], or philodendron (Philodendron Schott.), but areca palm [Dypsis lutescens (H. Wendl.) Beentje \& J. Dransf.] shoot dry weight was higher under overhead than ebb-and-flow irrigation. Ebb-and-flow irrigation was reported to be more water-use efficient (total plant dry weight per L water) than manual, drip, or capillary mat irrigation systems for growth of containerized poinsettias (Euphorbia pulcherrima Willd. Ex. Klotzsch) (Dole et al., 1994).

'Van Houttei' and S. guaranitica plants irrigated by ebb and flow had higher leaf $\mathrm{K}$ content and did not differ in leaf $\mathrm{N}$ or $\mathrm{Fe}$ content than plants irrigated manually or by drip. Blom and Piott (1992) reported that chrysanthemums (Dendranthema $\times$ grandiflorum) grown using ebb-and-flow irrigation had higher foliar N, K, Ca, Mg, Zn, and Fe contents than plants grown under drip irrigation. Percent water runoff was also reduced more with ebb-and-flow irrigation compared with drip or manual irrigation of poinsettia (Dole et al., 1994) and geranium (Pelargonium $\times$ hortorum L.H. Bailey) (Morvant et al., 1997).

Media moisture content measured 2, 4 and 6 weeks after transplanting was similar among irrigation systems but was highly influenced by media in each experiment (data not presented). This suggests that similar water contents were achieved throughout each experiment, regardless of water delivery method, frequency of application, or volume. However, the peat-based medium had a significantly higher moisture content than the 50\% or $100 \%$ compost medium each time measurements were taken (2, 4 and 6 weeks) for each experiment (data not presented). The higher bulk and particle densities of the compost medium (Table 1) may be associated with lower water retention than the $100 \%$ peat-based medium, regardless of the irrigation system. However, in 'Van Houttei', the lower moisture content of the compost amended medium (throughout the experiment) may have contributed to the slightly reduced plant growth characteristics, compared with plants grown in the peat-based medium.

In this study, plants grown in compost alone had similar growth for two out of the three salvia species than plants grown in the peat-based medium. However, all media and irrigation systems produced marketable plants for each salvia except for 'Van Houttei' grown in 50\% compost using drip irrigation. Compost may therefore be a viable complete or partial substitute for peat in commercial mixes used to produce 'Indigo Spires', S. gauranitica, and 'Van Houttei' under manual, drip, or ebb-and-flow irrigation systems without a deleterious impact on plant appearance, marketable quality, or consumer acceptability. Beneficial plant growth and development has been reported for marigold (Tagetes erecta L.), zinnia (Zinnia elegans Jacq.) and petunia (Petunia hybrida Hort.) (Wootton et al., 1981), impatiens (Impatiens wallerana Hook.) (Klock and Fitzpatrick, 1997), and pansy (Viola tricolor L.) and snapdragon (Antirrhinum majus L.) (Hemphill et al., 1984) grown in media with biosolid-derived compost.

In the past, variation within and between commercial compost facilities reduced the quality of compost for horticultural enterprises. Potential hazards of compost utilization have been heavy metal contents (particularly with the use of biosolids), high salt content (particularly with foodscrap feedstock), and phytotoxicity associated with sensitive plant species (Stoffella and Kahn, 2001). Horticultural grade composts of sufficient quantity and quality are now being produced by private enterprises and public municipalities. Therefore, composts may be a viable partial substitute for peat use in containerized nursery media, while maintaining sufficient plant growth, development, and ultimately plant quality. Bugbee et al. (1991) also concluded that growth was similar or greater for aster (Asternovi-belgii), whirling butterflies (Gaura lindheimeri), or sedum (Sedum purpureum) cuttings grown in biosolid-derived compost. Due to the limited supply and escalating costs of peat (Barkham, 1993) combined with the beneficial properties of compost (Stoffella and Kahn, 2001) and similar plant growth and quality response to compost amended media, compost should be an attractive alternative for the production of subtropical perennials under diverse irrigation systems.

\section{Literature Cited}

Barkham, J.P. 1993. For peat's sake: Conservation or exploitation? Biodiv. Conser. 2:556-566.

Bernstein, L. 1975. Effects of salinity and sodicity on plant growth. Annu. Rev. Phytopathol. 13:295-312.

Blom, T.J. and B.D. Piott. 1992. Preplant moisture content and compaction of peatwool using two irrigation techniques on potted chrysanthemums. J. Amer. Soc. Hort. Sci. 117:220-223.

Bragg, N.C. and B.J. Chambers. 1988. Interpretation and advisory applications of compost air-filled porosity (AFP) measurements. Acta Hort. 221:35-44.

Bremner, J.M. 1996. Nitrogen-Total, p. 1085-1122. In: D.L. Sparks, A.L. Page, P.A. Helmke, and R.H. Loeppert (eds.). Methods of soil analysis. Part 3 - Chemical methods. Soil Sci. Soc. Amer. Book Ser. 5. Bugbee, G.J., C.R. Frink, and D. Migneault. 1991. Growth of perennials and leaching of heavy metals in media amended with a municipal leaf, sewage sludge and street sand compost. J. Environ. Hort. 9:47-50.

Chase, A.R. and C.A. Conover. 1993. Algae control in an ebb and flow irrigation system. Proc. Fla. State Hort. Soc. 106:280-282.

Clebsch, B. 2001. Salvias cross the border. Horticulture 98(5):51-53.

Conover, C.A. and J.N. Joiner. 1966. Garbage compost as a potential soil component in production of Chrysanthemum morifolium 'Yellow Delaware' and 'Oregon'. Proc. Fla. State Hort. Soc. 79:424-426.

D’Angelo, G., M. Pusterla, and M. Castelnuovo. 1995. Response of peatand compost-based substrates to different levels of irrigation and fertilization in cyclamen. Acta Hort. 401:537-544.

Davidson, H., R. Mecklenburg, and C. Peterson. 1994. Nursery management: Administration and culture. $3^{\text {rd }}$ ed. Prentice Hall, Englewood 
Cliffs, N.J.

Dole, J.M., J.C. Cole, and S.L. von Broembsen. 1994. Growth of poinsettias, nutrient leaching, and water-use efficiency respond to irrigation methods. HortScience 29:858-864.

Fitzpatrick, G.E. 2001. Compost utilization in ornamental and nursery crop production systems, p. 135-150. In: P.J. Stoffella and B.A. Kahn. (eds.). Compost utilization in horticultural cropping systems. Lewis Publ., Boca Raton, Fla.

Fitzpatrick, G.E., E.R. Duke, and K.A. Klock-Moore. 1998. Use of compost products for ornamental crop production: Research and grower experiences. HortScience 33:941-944.

Gartner, J.B., S.M. Still, and J.E. Klett. 1974. The use of bark waste as a substrate in horticulture. Acta Hort. 37:2003-2012.

He, Z., X. Yang, B.A. Kahn, P.J. Stoffella, and D.V. Calvert. 2001. Plant nutrition benefits of phosphorus, potassium, calcium, magnesium, and micronutrients from compost utilization, p. 307-320. In: P.J. Stoffella and B.A. Kahn (eds.). Compost utilization in horticultural cropping systems. Lewis Publ., Boca Raton, Fla.

Hemphill, D.D. Jr., R.L. Ticknor, and D.J. Flower. 1984. Growth response of annual transplants and physical and chemical properties of growing media as influenced by composted sewage sludge amended with organic and inorganic materials. J. Environ. Hort. 2:112-116.

Hoitink, H.A.J., Y. Inbar, and M.J. Boehm. 1991. Status of compostamended potting mixes naturally suppressive to soil-borne diseases of floricultural crops. Plant Dis. 75:869-873.

Hue, N.V. and B.A. Sobieszczyk. 1999. Nutritional values of some biowastes as soil amendments. Compost Sci. Utiliz. 7(1):34-41.

Inbar Y., Y. Chen, and H.A.J. Hoitink. 1993. Properties for establishing standards for utilization of compost in container media, p. 668-694. In: H.A.J. Hoitink and H.M. Keener (eds.). Science and engineering of composting: Design, environmental, microbiological and utilization aspects. Renaissance Publ., Worthington, Ohio.

Joiner, J.N. 1981. Foliage plant production. Prentice-Hall, Englewood Cliffs, N.J.

Klock, K.A. and G.E. Fitzpatrick. 1997. Growth of impatiens 'Accent Red' in three compost products. Compost Sci. Utiliz. 5(4):26-30.

Klock-Moore, K.A. and T.K. Broschat. 2001. Effect of four growing substrates on growth of ornamental plants in two irrigation systems. HortTechnology 11:456-460.

Lumis G.P. and A.G. Johnson. 1982. Boron toxicity and growth suppression of Forsythia and Thuja grown in mixes amended with municipal waste compost. HortScience 17:821-822.

Morvant, J.K., J.M. Dole, and E. Allen. 1997. Irrigation systems alter distribution of roots, soluble salts, nitrogen, and $\mathrm{pH}$ in the root medium. HortTechnology 7:156-160.
Nelson, D.W. and L.E. Sommers. 1996. Total carbon, organic carbon, and organic matter, p. 961-1010. In: D.L. Sparks, A.L. Page, P.A. Helmke, and R.H. Loeppert (eds.). Methods of soil analysis. Part $3-$ Chemical methods. Soil Sci. Soc. Amer., Book Ser. 5.

Niedziela, C.E. and P.V. Nelson. 1992. A rapid method for determining physical properties of undisturbed substrate. HortScience 27:12791280.

Poole, R.T., C.A. Conover, and J.N. Joiner. 1981. Soils and potting mixtures, p. 179-202. In: J.N. Joiner (ed.). Foliage plant production. Prentice Hall, Englewood Cliffs, N.J.

Sanderson, K.C. 1980. Use of sewage-refuse compost in the production of ornamental plants. HortScience 15:173-178.

SAS Institute Inc. 1989. SAS/STAT user's guide. version $6.4^{\text {th }}$ ed. vol. 2. SAS Inst., Cary N.C.

Shiralipour, A., B. Faber, and M. Chrowstowski. 1996. Greenhouse broccoli and lettuce growth using co-composted biosolids. Compost Sci. Utiliz. 4:38-43.

Sims, J.T. 1995. Organic wastes as alternatives nitrogen sources, p. 487535. In: P.E. Bacon (ed.). Nitrogen fertilization in the environment. Marcel Dekker, New York.

Staff writer. 2001. Introducing the 2001 Florida plants of the year. Ornamental Outlook 9(11):17-22.

Stoffella, P.J. and B.A. Kahn (eds.). 2001. Compost utilization in horticultural cropping systems. Lewis Publ., Boca Raton, Fla.

United States Environmental Protection Agency (USEPA). 1994. A plain English guide to the EPA Part 503 Biosolids Rule. EPA/832/R93/003. Office Wastewater Mgt., Wash., D.C.

Uva, W.L., T.C. Weiler, and R.A. Milligan. 1998. A survey on the planning and adoption of zero runoff subirrigation systems in greenhouse operations. HortScience 33:193-196.

Vavrina, C.S. 1994. Municipal solid waste materials as soilless media for tomato transplant production. Proc. Fla. State Hort. Soc. 107:118-120.

Wilson, S.B., P.J. Stoffella, and D.A. Graetz. 2001. Compost-amended media for growth and development of mexican heather. Compost Sci. Utiliz. 9(1):60-64.

Wilson, S.B., P.J. Stoffella, and D.A. Graetz. 2002. Production of three perennial species using organic-based media. Sci. Hort. 93:311-320.

Wootton, R.D., F.R. Gouin, and F.C. Stark. 1981. Composted, digested sludge as a medium for growing flowering annuals. J. Amer. Soc. Hort. Sci. 106:46-49.

Zucconi, F. and M. de Bertoldi. 1991. Specifications for solid waste compost, p. 200-205. In: The staff of Biocycle (eds.). The Biocycle guide to the art and science of composting. JG Press, Inc. Emmaus, Pa.

Zucconi, F., A. Pera, M. Forte, and M. de Bertoldi. 1981. Evaluating toxicity of immature compost. Biocycle 22:54-57. 